\title{
Caso clínico: Síndrome de Ogilvie.
}

\section{Clinical Case: Ogilvie Syndrome.}

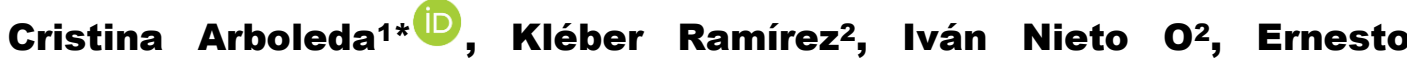 Paladines².}

1. Universidad Estatal de Guayaquil, Facultad de Ciencias Médicas, Departamento de Postgrados.

*Correspondencia:

crisalex48@hotmail.com

Teléfono [593] 989392891

Conflicto de intereses: Los autores declaran no tener conflictos de intereses.

Fondos: Ver la página 193

Recibido: 19 Enero 2017

Aceptado: 5 Diciembre 2017

Publicado: 30 Diciembre 2017

Membrete bibliográfico:

Arboleda C, Ramírez K, Nieto I, Paladines E. Caso clínico: Sïndrome de Ogilvie. Rev. Oncol. Ecu 2017;27(3):189-194.

DOI: https://doi.org/10.33821/230

Copyright Arboleda, et al. Este artículo es distribuido bajo los términos de Creative Commons Attribution License, el cual permite el uso y redistribución citando la fuente y al autor original.
2. Instituto Oncológico Nacional "Dr Juan Tanca Marengo", Solca-Guayaquil, Servicio de Gastroenterología.

\section{Resumen}

Introducción: El síndrome de Ogilvie es una patología rara que se puede encontrar en pacientes que han sido sometidos a cirugías extensas dando un cuadro clínico de obstrucción intestinal, llegando a ser letal de no ser diagnosticada a tiempo. Existen varios tratamientos tanto farmacológicos, endoscópicos y quirúrgicos para descomprimir el colon.

Caso clínico: Se presenta el caso de un paciente de 35 años intervenido quirúrgicamente de un meningioma que sufre de un cuadro de obstrucción intestinal posterior a la intervención catalogándose como un Síndrome de Ogilvie.

Palabras Claves: SEUDOOBSTRUCCIÓN INTESTINAL, CECOSTOMÍA.

DOI: $10.33821 / 230$

\section{Abstract}

Introduction: Ogilvie syndrome is a rare condition that can be found in patients who have undergone extensive surgery giving a clinical picture of intestinal obstruction, becoming lethal if not diagnosed in time. There are several pharmacological, endoscopic and surgical treatments to decompress the colon.

Clinical case: We present the case of a 35 year-old patient surgically operated on a meningioma suffering from a picture of intestinal obstruction following the intervention, being classified as an Ogilvie Syndrome. The case is presented by the low incidence of the same.

Keywords: INTESTINAL SEUDOOBSTRUCTION, CECOSTOMY.

DOI: $10.33821 / 230$ 


\section{Introducción}

El síndrome de Ogilvie fue descrito en 1948 por el doctor William Ogilvie [1] en laparotomías exploratorias de pacientes con masas retroperitoneales con invasión del plexo celíaco [2]. La fisiopatología aún no se ha identificado pero podría ser por una alteración motriz del colon. Los pacientes más afectados de este síndrome son varones, encamados y con antecedentes de cirugías amplias [3].

Este síndrome se caracteriza por distensión abdominal predominantemente del colon del lado derecho [4]; de manera aguda 24 - 72 horas, con timpanismo generalizado, acompañado de deposiciones líquidas, se puede auscultar ruidos hidroaéreos, sin embargo hay casos en los que hay dificultad para canalizar flatos. Al inicio del cuadro clínico no hay signos de peritonismo [5] y los recuentos de leucocitos están dentro de parámetros normales. Se debe considerar en pacientes que no se evidencia una obstrucción mecánica.

El propósito del presente es dar a conocer este raro síndrome asociado a cirugías de larga duración.

\section{Caso clínico}

Se trata de paciente varón de 35 años intervenido quirúrgicamente de meningioma en base del cráneo que luego de su cirugía es transferido al servicio de Terapia Intensiva para mantenerse bajo sedación. Luego de 4 días de su intervención, desarrolla neumonía asociada al ventilador donde se aisla Klebsiella Pneumoniae resistente a carbapenémicos. Desde las 72 horas post-quirúrgicas se observa leve distensión del abdomen. Se realiza tomografía axial computarizada abdomino pélvica a las 96 horas encontrándose distensión de colon derecho hasta ángulo esplénico de $12 \mathrm{~cm}$ aproximadamente y dilatación del intestino delgado (Figuras 1 y 2 2). Se decide iniciar tratamiento farmacológico con metoclopramida y eritromicina como procinéticos. Al no obtener mejoría es intervenido quirúrgicamente realizándose colostomía de descarga, observando, como hallazgos quirúrgicos, signos de sufrimiento intestinal no se realiza resección del asa por las condiciones del paciente. Paciente con mala evolución clínica, que luego de 10 días del diagnóstico fallece por insuficiencia respiratoria aguda secundaria a fallo orgánico multisistémico. 
Figura 1. Tomografía computarizada en corte coronal donde se observa dilatación de colon derecho más dilatación de asas de intestino delgado.

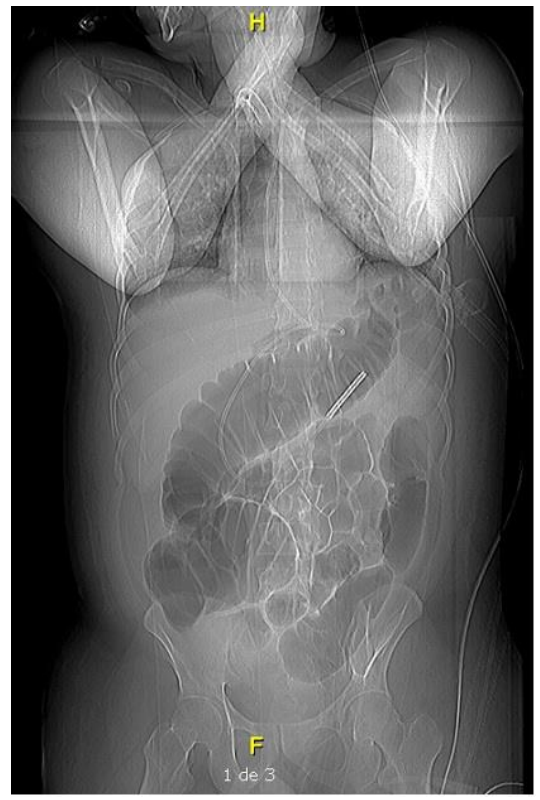

Figura 2.- Tomografía computarizada con contraste oral (hidrosoluble) corte axial se observa gran dilatación de colon derecho

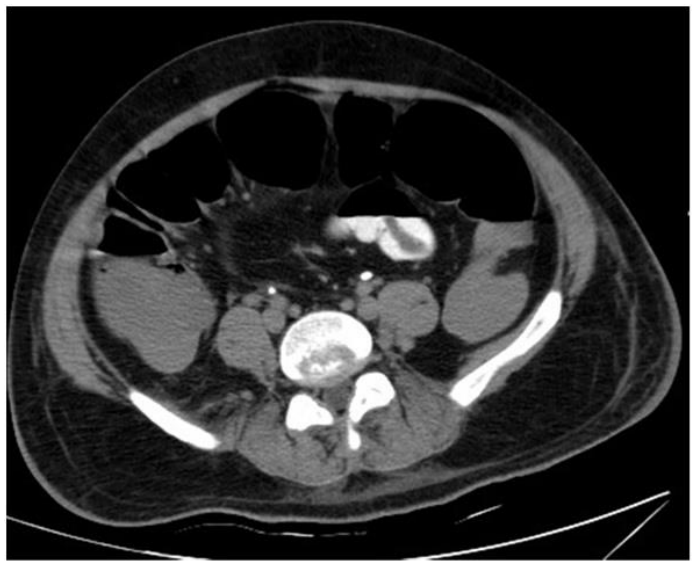

\section{Discusión}

No se conoce con exactitud la prevalencia del Síndrome de Ogilvie sin embargo se sabe que es escasa. Se caracteriza por dolor abdominal y distensión la cual simula una obstrucción intestinal [6]. Para el diagnóstico se necesita una radiografía de abdomen, sin embargo, por la alta probabilidad de perforación es conveniente solicitar una tomografía. Se puede encontrar dilatación del ciego, colon ascendente y transverso; en varias ocasiones se puede encontrar dilatación del intestino delgado. 
Su diagnóstico temprano ayuda a reducir la morbilidad y mortalidad en un $80 \%$ [7].

El tratamiento del Síndrome de Ogilvie es farmacológico con medicamentos procinéticos como la neostigmina [8] $2 \mathrm{~g}$ intravenoso tomando en cuenta las posibles complicaciones; se puede utilizar eritromicina entre otros. La descompresión endoscópica tras el fallo farmacológico [9], se debe realizar con colonoscopios de doble canal para la eliminación del gas y de las heces. Además se podría colocar un tubo de drenaje con ayuda del fluoroscopio. En el tratamiento quirúrgico debe realizarse una cecostomía percutánea [10].

Su diagnóstico diferencial se debe hacer con la pseudoosbtrucción aguda del colon y con el megacolon tóxico secundario a clostridium difficile [11]. El paciente tenía los siguientes factores asociados: craniotomía, uso de corticoides alta dosis, uso de benzodiacepinas, neumonía.

Dentro de sus complicaciones se puede encontrar la isquemia y perforación [12], se ha considerado que un diámetro cecal mayor de $12 \mathrm{~cm}$ está relacionado con la perforación [13] y debe sospecharse cuando hay neumoperitoneo y los recuentos de leucocitos han aumentado. Tal como la leucocitosis asociada a la coinfección por klebsiella pneumoniae presentada en este paciente, lo que no permitió una buena respuesta al tratamiento dado. Este diagnóstico en este paciente se estableció por la distensión abdominal aguda luego de la neurocirugía, el abdomen estaba timpánico, con dilatación de colon derecho y las asas del intestino delgado, recuento leucocitario elevado además se realizó citología del moco rectal descartando la infección por clostridium difficile, se han descartado así mismo obstrucciones mecánicas y anomalías electrolíticas.

\section{Conclusiones}

Se debe considerar este síndrome cuando exista dilatación del colon derecho y el paciente haya sido sometido a una cirugía grande o ha permanecido encamado por largo tiempo.

\section{Agradecimientos}

Queremos agradecer a los familiares del paciente por autorizar la publicación del presente caso.

\section{Información adicional}

Nota del Editor

La Revista Oncología Ecu

permanece neutral con

respecto a los reclamos jurisdiccionales en mapas publicados y afiliaciones institucionales.

\section{Abreviaturas}

Ninguna

Archivos Adicionales

Ninguno declarado por los autores. 
Fondos

Los fondos de la investigación fueron propios de los autores del presente artículo.

\section{Disponibilidad de datos y materiales}

Existe la disponibilidad de datos bajo solicitud al autor de correspondencia. No se reportan otros materiales.

\section{Contribuciones de los autores}

$\mathrm{CA}, \mathrm{KR}$, IN idea de investigación, EP, CA revisión bibliográfica, compilación del caso, escritura del artículo. IN realizó el análisis crítico del artículo. Todos los autores leyeron y aprobaron la versión final del artículo.

Aprobación de ética y consentimiento para participar

No aplica a un caso clínico.

\section{Consentimiento para publicación}

Los autores cuentan con el consentimiento escrito para publicación por parte de los familiares del paciente.

\section{Información de los autores}

Cristina Arboleda, Médica postgradista de Gastroenterología. Facultad de Ciencias Médicas de la Universidad Estatal de Guayaquil. (D) https://orcid.org/0000-00017496-0053

Kléber Ramírez, Médico tratante de Gastroenterología. Instituto Oncológico Nacional "Dr Juan Tanca Marengo", Solca-Guayaquil, Servicio de Gastroenterología.

Iván Nieto 0, Jefe del servicio de gastroenterología. Instituto Oncológico Nacional "Dr Juan Tanca Marengo", Solca-Guayaquil, Servicio de Gastroenterología.

Ernesto Paladines, Médico tratante de Gastroenterología. Instituto Oncológico Nacional "Dr Juan Tanca Marengo", Solca-Guayaquil, Servicio de Gastroenterología. 


\section{Referencias}

Abreviaturas en la referencias

DOI: Digital Object

Identifier

PMID: PubMed Identifier

SU: Short URL
1. Rendón M. Síndrome dismórfico neonatal y síndrome de Ogilvie. Cir y Cir. 2017;85(2):148-153. DOI: 10.1016/j.circir.2015.11.004

2. Pereira $P$, Djeudji $F$, Leduc $P$, Fanget $F$, Barth $X$. Ogilvie's syndrome - Acute colonic pseudo-obstruction. J Chir Viscerale. 2015;152(2):99-106. DOI: 10.1016/j.jviscsurg.2015.02.004

3. Martins L, Borges T, Magnago de Mattos A, Martins R, Carvalho M, Kalil M. SíNDROME DE OGILVIE SEGMENTAR - DESAFIO DIAGNÓSTICO. J. Coloproctol. 2018; 38(2): 1-192. DOI: 10.1016/j.jcol.2018.08.175

4. Nadukkandiyil N, Alhamad HK, Wahab LA, Al Sulaiti EM, Sankaranarayanan A. Acute intestinal pseudoobstruction (Ogilvie's syndrome): A case report. J Clin Gerontol Geriatr. 2014;5(4):1-3. DOI: 10.1016/j.jcgg.2014.02.003

5. Keller J, Layer P. Akute Kolonpseudoobstruktion: Ogilvie-Syndrom. Medizinische Klin - Intensivmed und Notfallmedizin. 2015;110(7):506-509. DOI: 10.1007/s00063-015-0081-4

6. Ogilvie H. Large-Intestine Colic Due To Sympathetic Deprivation A New Clinical Syndrome. Br Med J. 1948;2(4579):671-673. DOI: 10.1136/bmj.2.4579.671

7. Walwaikar PP, Kulkarni SS, Bargaje RS. Evaluation of new gastro-intestinal prokinetic (ENGIP-II) study $\mathrm{J}$ of the Indian Med Assoc. 2005; 103(12): 708-709. PMID: 16821670.

8. Kayani B, Spalding DR, Jiao LR, Habib NA, Zacharakis E. Does neostigmine improve time to resolution of symptoms in acute colonic pseudo-obstruction?. Int J Surg. 2012;10(9):453-457. DOI: 10.1016/j.ijsu.2012.08.008

9. Tsirline VB, Zemlyak AY, Avery MJ, Colavita PD, Christmas AB, Heniford BT, et al. Colonoscopy is superior to neostigmine in the treatment of Ogilvie's syndrome. Am J Surg. 2012;204(6):849-855. DOI: 10.1016/j.amjsurg.2012.05.006

10. Mesa JL, Pablos MS. Pseudoobstrucción aguda del colon o síndrome de Ogilvie y evisceración. Rev Cub Cir. 2016;55(1):85-91. SU: goo.gl/iL47jG

11. Cebola M, Eddy E, Davis S, Chin-Lenn L. Acute colonic pseudo-obstruction (Ogilvie's syndrome) following total laparoscopic hysterectomy. J Minim Invasive Gynecol. 2015;22(7):1307-1310. DOI: 10.1016/j.jmig.2015.06.023

12. Kaiser AM. Ogilvie transition to colonic perforation. Am J Surg. 2010;200(1):e15-e16. DOI: 10.1016/i.amjsurg.2009.10.029

13. Riesco JM, Manzano R. Síndrome de Ogilvie. Rev. esp. enferm. dig. 2013; 105( 3 ): 175-175. Dol: $10.4321 / \mathrm{S} 1130-01082013000300011$ 